\title{
Assessment of Apoptosis Level of Naive CD8+T-1ymphocytes in Children with Acute Infectious Mononucleosis in CD95 and DR3 Receptors Activation
}

DOI: $10.17691 / \operatorname{stm} 2015.7 .3 .16$

Received April 13, 2015

E.N. Filatova, PhD, Leading Researcher, Laboratory of Molecular Biology and Biotechnology :

O.V. Utkin, PhD, Head of Laboratory of Molecular Biology and Biotechnology ${ }^{1}$; Associate Professor,

Department of Microbiology and Immunilogy2;

E.V. Anisenkova, Junior Researcher, Laboratory of Molecular Biology and Biotechnology";

N.B. Presnyakova, Researcher, Laboratory of Molecular Biology and Biotechnology';

T.D. Sycheva, Resident, Department of Children Diseases ${ }^{2}$;

V.V. Krasnov, MD, DSc, Professor, Head of the Department of Children Diseases;

N.E. Senyagina, MD, PhD, Associate Professor, Department of Children Diseases²;

E.A. Kulova, MD, PhD, Tutor, Department of Children Diseases²;

E.I. Efimov, MD, DSc, Professor, Director ${ }^{1}$; Head of the Department of Microbiology and Immunology ${ }^{2}$

${ }^{1}$ Blokhina Research Institute of Epidemiology and Microbiology of Federal Service for Surveillance on Consumer Rights Protection and Human Wellbeing, 71 Malaya Yamskaya, Nizhny Novgorod, 603950, Russian Federation;

${ }^{2}$ Nizhny Novgorod State Medical Academy, 10/1 Minin and Pozharsky Square, Nizhny Novgorod, 603005,

Russian Federation

The aim of the investigation was to estimate the relation of CD95 and DR3 receptors activation with apoptosis level of naive cytotoxic T-lymphocytes ( $\mathrm{nCTL}$ ) in children with acute infectious mononucleosis (AIM).

Materials and Methods. The test materials were peripheral blood samples of healthy children and children with AIM. nCTL were isolated by negative immunomagnetic separation. Specific activation of CD95 and DR3 receptors was performed using monoclonal antibodies. An apoptosis level and expression of receptors were studied by flow cytometry.

Results. The percentage of cell apoptosis decreased in children with AIM in freshly isolated nCTL, as well as in CD95 receptor activation compared to healthy children. nCTL apoptosis in healthy children regardless of culture conditions was accompanied by the reduced quantity of CD95+DR3- cells and CD95 expression density on their surface. In children with AIM the decrease of these indices required CD95 activation. Compared to healthy children, the percentage of CD95+DR3 ${ }^{+}$cells in children with AIM decreased in CD95 activation. In CD95 receptor activation in healthy children and children with AIM, the content of CD95+DR3 ${ }^{+}$cells correlated directly with an apoptosis level. DR3 receptor activation was accompanied neither by $\mathrm{nCTL}$ apoptosis level change nor the changed content of DR3 ${ }^{+}$cells in both healthy children and children with AIM.

Conclusion. $\mathrm{nCTL}$ are less sensitive to apoptosis in children with AIM compared to healthy children. DR3 receptor activation results in no change of $\mathrm{nCTL}$ apoptosis level both in healthy children and children with AIM. CD95 activation in patients with AIM is accompanied by increased resistance of CD95+DR3- cells to apoptosis and the susceptibility to apoptosis of CD95+DR3 ${ }^{+}$cells. The evaluation of nCTL susceptibility to CD95-induced apoptosis in AIM can serve as a subtest to assess the state of a cell component of immune system.

Key words: CD95; DR3; apoptosis; naive cytotoxic T-lymphocytes; acute infectious mononucleosis; death receptors.

Acute infectious mononucleosis (AIM) is a widespread viral disease. Its agents are Herpesviridae: Epstein-Barr virus (EBV), cytomegalovirus (CMV), type 6 human herpes virus. Over $90 \%$ of urban population are virus carriers. The disease is characterized by life-long latent virus persistence and its intermittent reactivation in a host. Only $10 \%$ of infected subjects develop an acute form of the disease, it occurring most frequently in childhood [1, 2].

AIM agents are lymphotropic viruses, which influence the functional state of immune system cells, e.g., they cause anergy of T-lymphocytes and regulate proliferation and apoptosis of immune competent cells [3].

The representatives of a protein family of "death receptors" - CD95 (Fas) and DR3 (LARD) - are involved in apoptosis initiation and cell proliferation. They express on the surface of immune competent cells including $\mathrm{CD}^{+}$and $\mathrm{CD}^{+}$T-lymphocytes [4-8]. In the norm, T-lymphocytes respond differently to the activation of these receptors. Activated T-lymphocytes after CD95 receptor stimulation are known to die due to apoptosis, while naive T-cells start proliferating. DR3 receptor

For contacts: Filatova Elena Nikolaevna, e-mail: filatova@nniiem.ru 
stimulation can be accompanied by the enhancement of both: proliferation and apoptosis of activated T-lymphocytes. Moreover, naive T-cells are characterized by the resistance to mediated DR3 apoptosis [7, 9-11].

Naive CD8 ${ }^{+}$T-cells are precursors of effector cytotoxic T-lymphocytes, which play an important role in antiviral immunity realization. Maintaining a sufficient amount of naive $\mathrm{CD} 8^{+} \mathrm{T}$-lymphocytes circulating in peripheral blood is of primary importance in an immune response [12]. The enhancement of naive T-lymphocyte death due to apoptosis potentially decreases the efficiency of immune reactions.

AIM is accompanied by a significant increase of the content of total fraction of $\mathrm{CD}^{+}$cytotoxic T-lymphocytes in peripheral blood [13]. Patients with chronic disease during a reactivation period are found to have their reduced content associated with a poor prognosis [14]. AIM patients have an increased CD95 receptor expression on the membrane of T-lymphocyte including total fraction of $\mathrm{CD}^{+} \mathrm{T}$-cells, and apoptosis enhancement [3]. At the same time, in vitro models have shown CMV to suppress CD95 expression and inhibit CD95-mediated apoptosis in infected human fibroblasts and in some other cell lines $[15,16]$. The functional role of DR3 receptor in AIM immunopathogenesis is poorly studied. Mice not expressing DR3 are known to be more susceptible to CMV infection due to insufficient activation of $\mathrm{CD}^{+}$T-lymphocytes as well [17]. The problem of CD95 and DR3 participation in apoptosis of naive cytotoxic T-lymphocytes (nCTLs) in AIM, as well as a prognostic value of the functional activity of these receptors is still unsettled.

The aim of the investigation was to estimate the relation of CD95 and DR3 receptors activation with apoptosis level of naive cytotoxic T-lymphocytes in children with acute infectious mononucleosis.

\section{Materials and Methods}

nCTLs culture preparation. The test materials were peripheral blood samples of healthy children and children with AIM aged from 9 to 16 . The study complies with the declaration of Helsinki (adopted in June, 1964 (Helsinki, Finland) and revised in October, 2000 (Edinburg, Scotland)) and was approved by the Ethics Committee of Nizhny Novgorod State Medical Academy. An informed consent to blood samples was obtained from all parents or foster parents. Mononuclear cells of peripheral blood were fractionated in accordance with density gradient of Histopaque $\left(\rho=1.077 \mathrm{~g} / \mathrm{cm}^{3}\right.$, Sigma, USA). nCTLs were isolated by negative immunomagnetic separation using commercial kits EasySep (Stemcell Technologies, Great Britain) according to the manufacturer's instructions. $\mathrm{nCTLS}$ isolation purity was assessed by flow cytometry using fluorescently labeled antibodies: CD3-PE, CD45RO-PE-Cy7, CD45RA-PerCP-Cy5.5 and CD8APC-eFluor780 (eBioscience, USA). Isolation purity was over $97 \%$ (Figure 1).

nCTLs culture and activation. Isolated nCTLs were cultured at a concentration of $1.10^{6}$ cells $/ \mathrm{ml}$ in RPMl1640 (PanEco, Russia) medium by adding $10 \%$ fetal calf serum (PAA Laboratories, Austria) and $2 \mathrm{mM} \mathrm{L-glutamine}$ (PanEco, Russia) at $37^{\circ} \mathrm{C}$ and $5 \% \mathrm{CO}_{2}$. The receptors were specifically activated by monoclonal antibodies (mAbs) against human receptors CD95 (isotype IgM, clone $\mathrm{CH}-11$, eBoiscience, USA) or DR3 (isotype lgG $\mathrm{k}$, clone JD3, Beckman Coulter, USA) at a concentration of $200 \mathrm{ng} / \mathrm{ml}$. Cells cultured without activators added were used as control.

Flow cytometry. We applied a flow cytometer BD

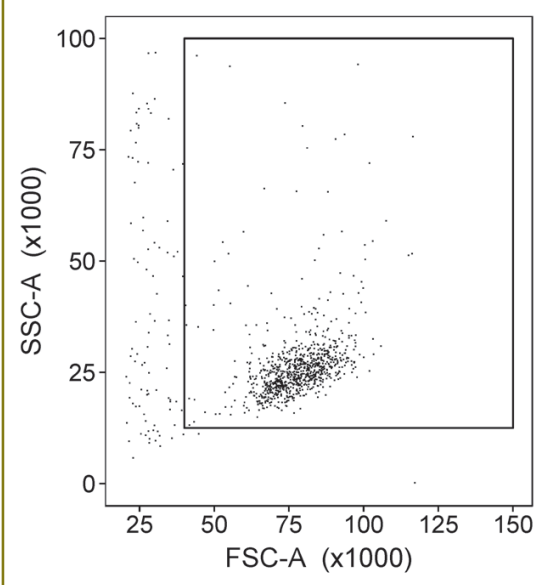

a

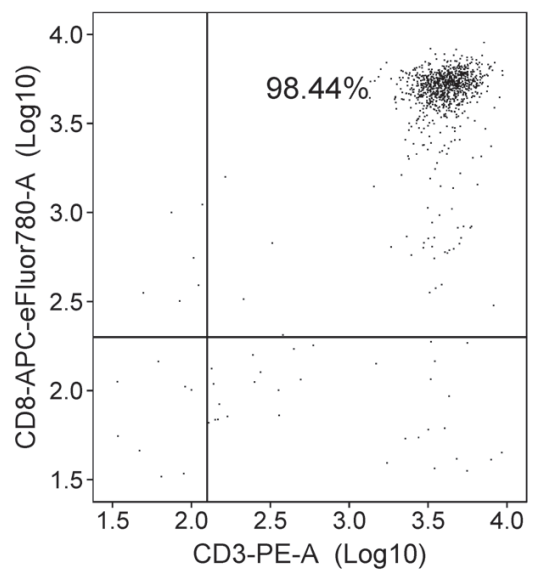

b

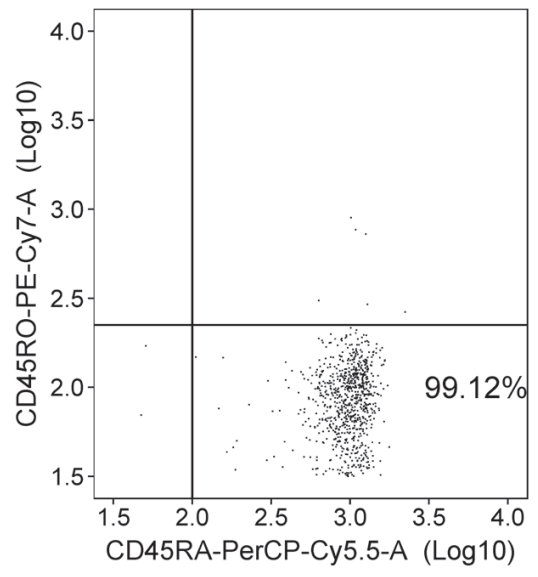

C

Figure 1. Control of nCTLs (naive cytotoxic T-lymphocytes) isolation purity by flow cytometry: based on forward and side scatter we separated debris and isolated lymphocyte gate $(a)$; in an isolated gate we analyzed the expression of CD3 and CD8 markers, the content of $\mathrm{CD}^{+} \mathrm{CD} 8^{+}$lymphocytes being over $98 \%$ (b); in $\mathrm{CD} 3^{+} \mathrm{CD} 8^{+}$lymphocyte pool we studied the expression of CD45RA and CD45RO markers, the content of CD45RA+CD45RO- cells being over $99 \%$ (c). The resulting content of naive $\mathrm{CD}^{+} \mathrm{T}^{+}$-lymphocytes was over $97 \%$ 
FACS Canto II (Becton, Dickinson and Company, USA) in the study. To normalize voltage on a photomultiplier we used gauge particles Cytometry setup and tracking beads (BD Biosciences, USA). The settings of fluorescence compensation were optimized by means of a commercial kit Anti-mouse lg, k/negative control compensation particles set (BD Biosciences, USA). A flash level for fluorescently labeled antibodies characterizing non-specific bonding was determined using corresponding isotypical controls. The data were collected using FACSDiva (BD Biosciences, USA). 3.104 cells were analyzed in each sample.

CD95 and DR3 expression and apoptosis assessment. Flow cytometry was used for the assessment. We analyzed newly isolated nCTLs, as well as nCTLs after 20-hour culture with activators or without them. For apoptosis assessment we applied double annexin V-PE $(A V)$ and 7-aminoactinomycin-D (7AAD) staining using a PE Annexin V Apoptosis Detection Kit (BD Biosciences, USA). The expression of CD95 and DR3 on blood lymphocyte membrane was analyzed by fluorescently labeled anti-CD95-PE-Cy7 (eBioscience, USA) and DR3-DyLight488 antibodies (Novus Biological, USA). Based on forward and side scatter we separated debris and isolated total nCTLs gate (Figure 2 (a)). Based on $A V$ and $7 A A D$ staining we isolated the gates of living lymphocytes (AV-7AAD-), lymphocytes at early $\left(A V^{+} 7 A A D^{-}\right)$and late $\left(A V^{+} 7 A A D^{+}\right)$apoptosis stages. Total apoptosis level was determined by the cumulative percent of cells at early and late apoptosis stages. Further, the total gate of living cells and early apoptotic cells were analyzed separately (Figure 2 (b)). Based on the expression of CD95 and DR3 receptors we isolated cells with three different phenotypes: CD95+DR3-, CD95-
$\mathrm{DR}^{+}$and $\mathrm{CD}^{+} 5^{+} \mathrm{DR} 3^{+}$(Figure 2 (c)). We calculated each phenotype cell percentage of the total amount of cells in a gate. Expression density of CD95 and DR3 on lymphocyte membrane was assessed relying on mean fluorescence intensity of receptor carrying cells (MFI).

Data processing. The algorithm of analysis was written in R language using RStudio 0.98.507 (RStudio, USA). In the study we applied Student t-test for dependent and independent samplings, Wilcoxon criterion for dependent and independent samplings, repeated measures ANOVA, Friedman test. A correlation coefficient was calculated using Pearson criterion ( $r$ ) and Spearman rank correlation $\left(r_{s}\right) . P$ value in multiple comparisons was corrected considering Holm-Bonferroni correction. The differences were considered significant if $p<0.05$. The results were represented indicating median, 25 and 75 percentiles.

\section{Results}

The effect of CD95 and DR3 receptor activation on nCTLs apoptosis level. Compared to healthy children, AIM children were found to have 1.9 decrease of apoptotic cells in newly isolated nCTLs $(p=0.023)$, and when activated by anti-CD95 antibodies the number decreased by 3.1 times $(p=0.046)$ (Figure 3). The activation of anti-DR3 antibodies, as well as the culture without activators had no effect on the amount of apoptotic nCTLs, it being no different in healthy children and children with AIM.

In healthy children the culture of nCTLs with antiCD95 and anti-DR3 antibodies, as well as that without activators resulted in the increase of apoptotic cells. Compared to newly isolated nCTLs, apoptosis level grew by 3.8 times $(p=0.005)$ in the control, when activated by anti-CD95 antibodies it increased by 3.0 times $(p=0.005)$,

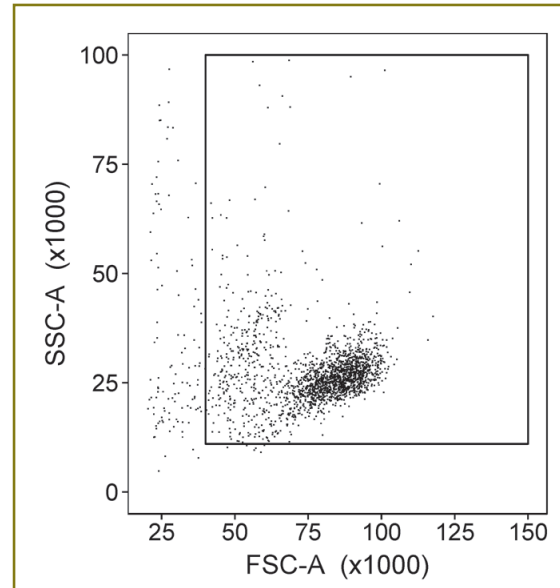

a

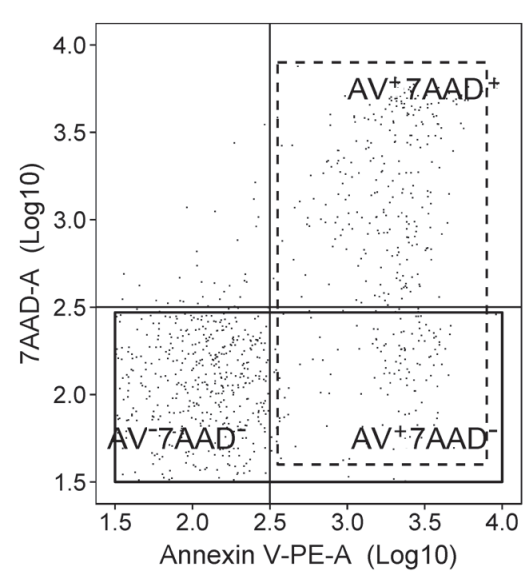

b

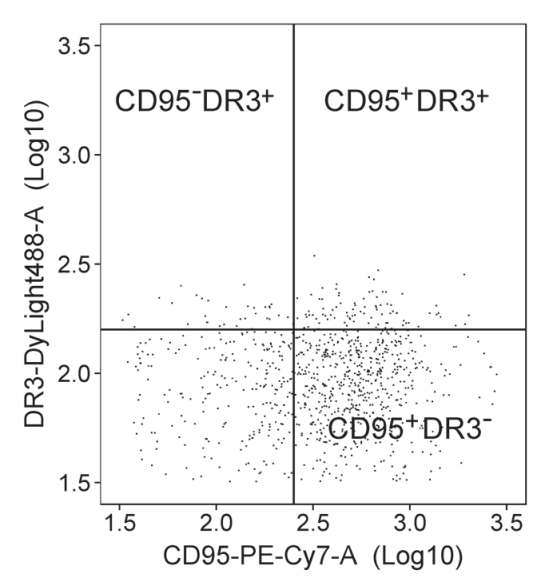

C

Figure 2. Gating principles when analyzing the expression of CD95 and DR3 and apoptosis level: lymphocyte gate was separated based on forward and side scatter (a); based on double AV and 7AAD staining, lymphocytes were divided into living cells, early and late apoptotic cells, the apoptosis level being determined as a total percentage of cells at early and late apoptosis stages (a dotted line). Living cells and early apoptotic cells were represented as a separate gate (a full line) (b); the expression of CD95 and DR3 receptors was studied in the gate (c) 


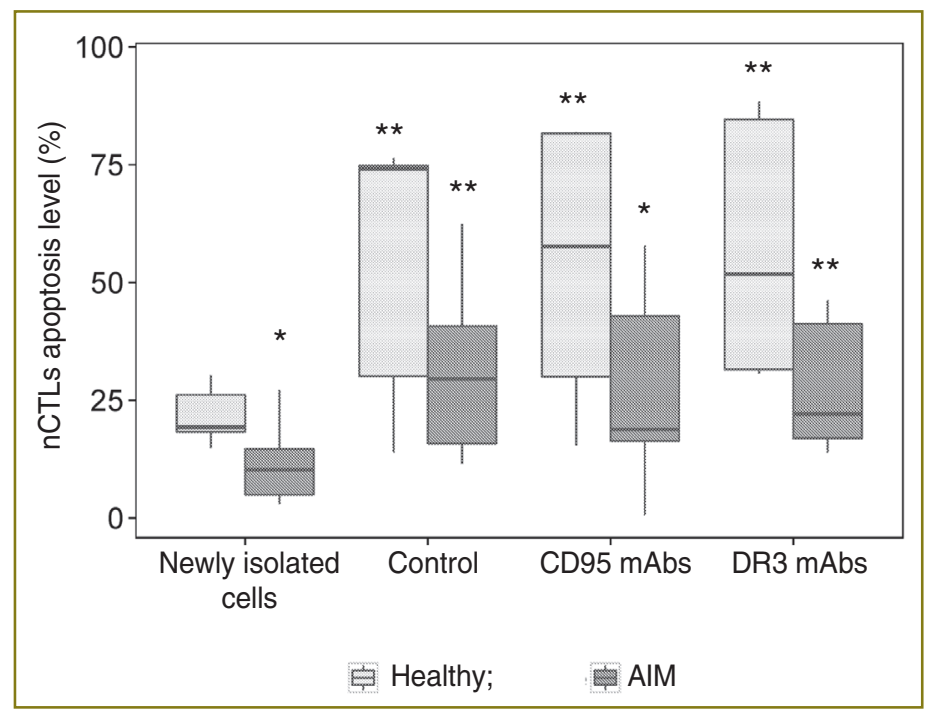

Figure 3. nCTLs (naive cytotoxic T-lymphocytes) apoptosis level. mAbs: monoclonal antibodies; AIM: acute infectious mononucleosis; * statistically significant differences compared to healthy children $(p<0.05)$; ${ }^{* *}$ compared to newly isolated $\mathrm{nCTLs}(\mathrm{p}<0.05)$

and when activated by anti-DR3 antibodies - by 2.7 times $(p=0.002)$. Children with AIM were found to have an increased number of apoptotic cells only in the control and when activated by anti-DR3 antibodies. Compared to newly isolated $\mathrm{nCTLs}$ the apoptosis level increased in the control by 2.9 times $(p=0.032)$, and after adding antiDR3 antibodies by 2.2 times $(\mathrm{p}=0.003)$.

Characteristic of $n C T L s$ with $C D 95^{+} D R 3^{-}$phenotype in healthy children and children with AIM. Children with AIM compared to healthy children were found to have no differences in the number of $\mathrm{CD}^{+} 5^{+} \mathrm{DR}^{-}$cells and CD95 expression density on their surface in newly isolated nCTLs, as well as when cultured, both in the control and when CD95 receptor was activated (Figure 4 and 5).

In healthy children, nCTLs culture compared to newly isolated cells was accompanied by the decrease of CD95+DR3- cell count and CD95 expression density on their surface in the control by 1.3 times $(p=0.034)$ and by 1.4 times $(p=0.018)$, when activated by antiCD95 antibodies - by 1.2 times $(p=0.034)$ and by 1.3 times $(p=0.013)$, respectively. AlM patients showed the decrease of $\mathrm{CD}^{+} 5^{+} \mathrm{DR}^{-}$cell number and CD95 expression density on their membrane only when $\mathrm{nCTLs}$ were stimulated by anti-CD95 antibodies - by 1.4 times $(p=0.001)$ and by 1.5 times $(p=0.001)$, respectively (See Figure 5).

Healthy children and children with AIM were found to have no correlation between nCTLs apoptosis level and the content of CD95 ${ }^{+}$DR3- cells, as well as CD95 expression density on their surfaces in all the cases under study.

Characteristic of nCTLs with CD95-DR3 ${ }^{+}$phenotype in healthy children and children with AIM. Children with AIM compared to healthy people showed a 5.1 decrease of CD95 $^{-}$DR3 $^{+}$cell number in newly isolated nCTLs and cultivated control cells $(p=0.013$ and $\mathrm{p}=0.038$, respectively). Moreover, DR3 expression density on the surface of $\mathrm{CD}^{-\mathrm{DR}}{ }^{+}$cells underwent no changes. Besides, both indices did not change when anti-DR3 antibodies were added

\section{(See Figure 4, Figure 6).}

Figure 4. Expression of CD95 and DR3 receptors on nCTLs (naive cytotoxic T-lymphocytes) surface. mAbs: monoclonal antibodies; AIM: acute infectious mononucleosis 


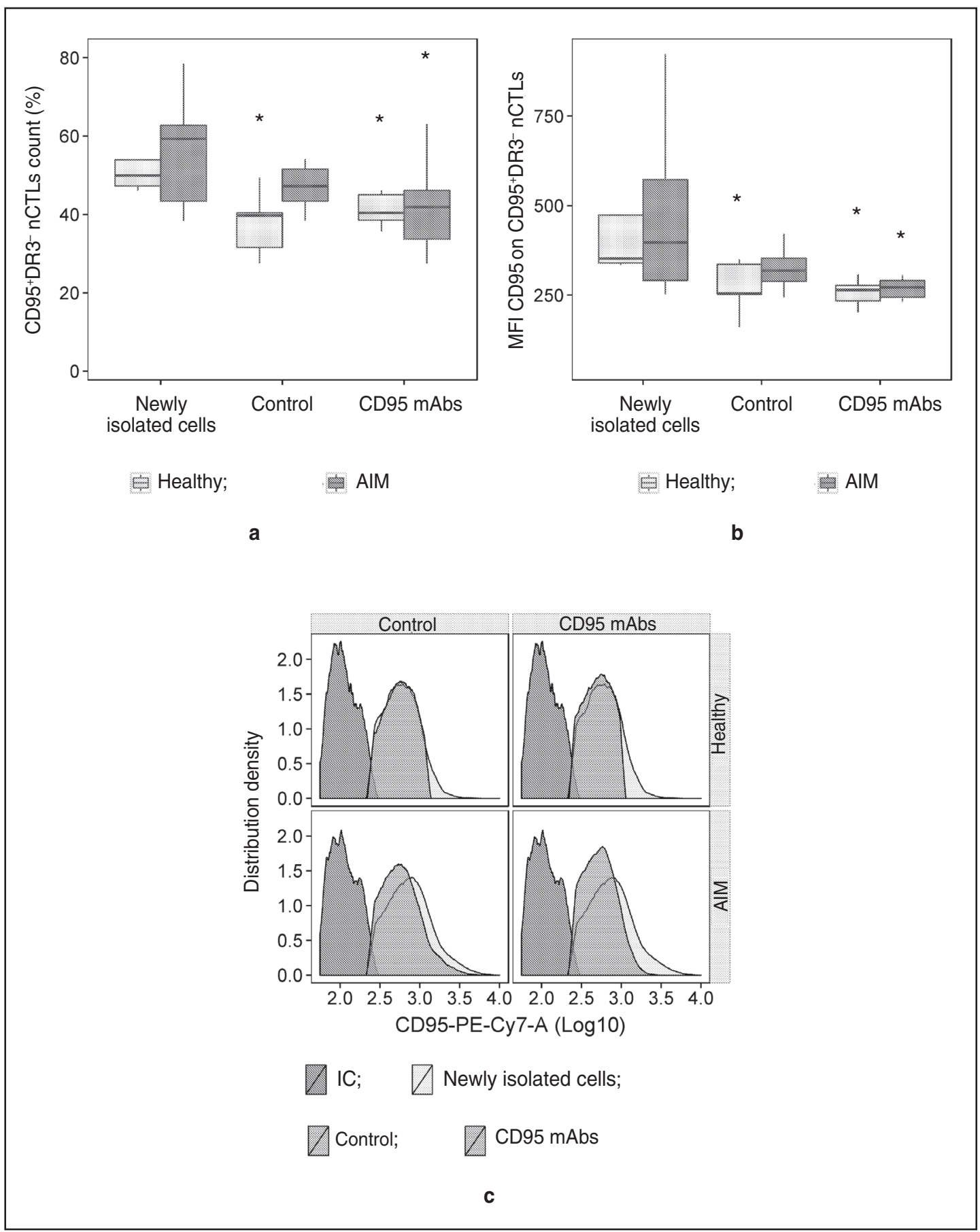

Figure 5. Characteristic of nCTLs (naive cytotoxic T-lymphocytes) with CD95+DR3- phenotype: the percentage of CD95+DR3- cells (a) and mean CD95 fluorescence intensity on their surface (b); the comparison of distribution density of CD95 receptor fluorescence on the surface of newly isolated CD95+DR3- nCTLs with the parameter when cultured without activators added or with anti-CD95 mAbs (monoclonal antibodies) added (c). AIM: acute infectious mononucleosis; IC: isotypical control; * statistically significant differences compared to newly isolated nCTLs $(p<0.05)$

Regardless of nCTLs culture conditions of healthy children and children with AIM, there were revealed no differences in the content of CD95-DR3 ${ }^{+}$cells and DR3 expression density on their surface compared to the newly isolated nCTLs (See Figure 6).

We found a direct correlation between the apoptosis level and the content of CD95-DR3 ${ }^{+}$nCTLs in healthy children and AIM children. In healthy children there was correlation when nCTLs were stimulated by anti-DR3 antibodies ( $r=0.93 ; p=0.023)$. Children with AIM showed the correlation of the indices under study in newly isolated $n C T L s(r=0.79 ; p=0.006)$. 


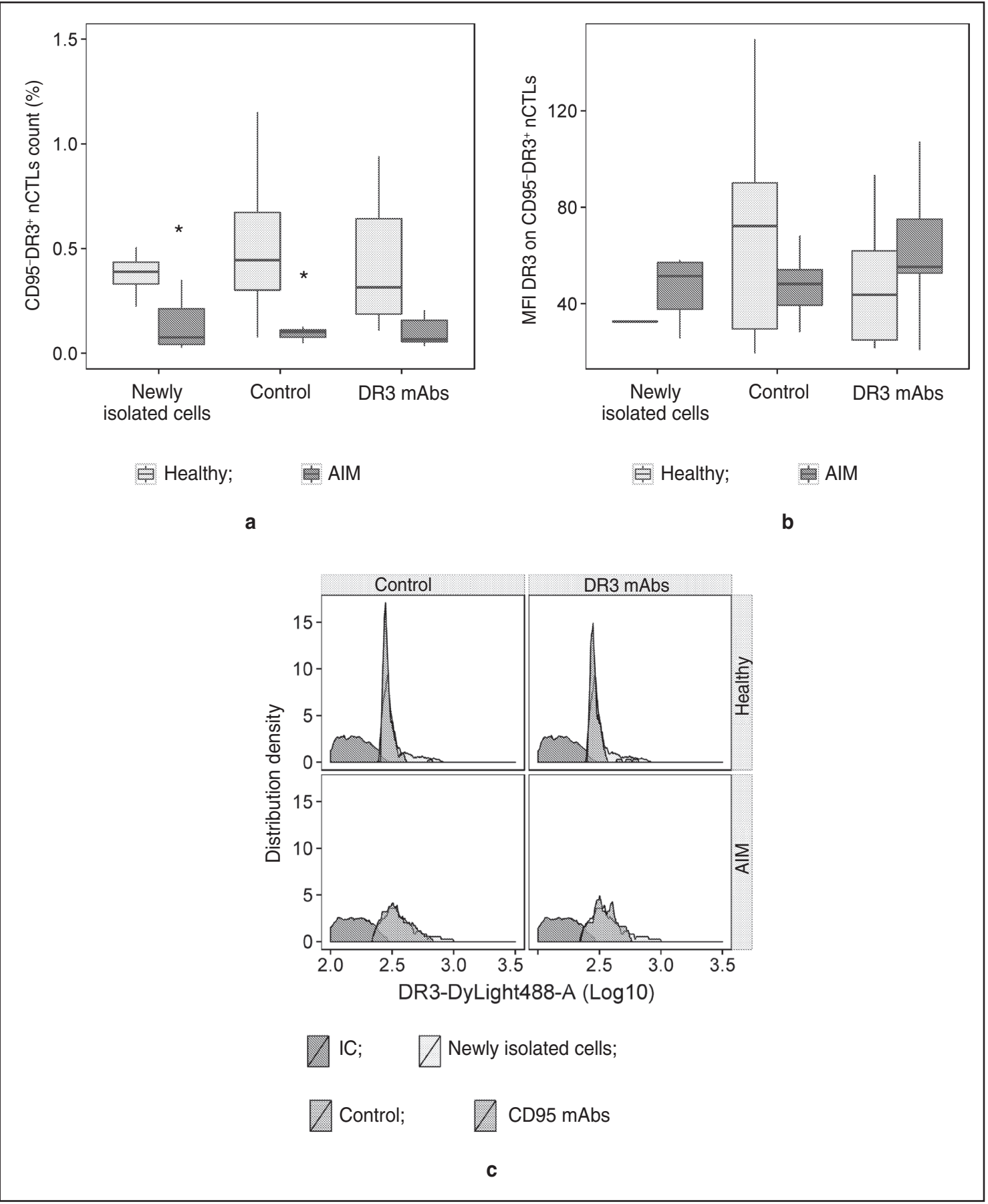

Figure 6. Characteristic of CD95-DR3 ${ }^{+}$nCTLs (naive cytotoxic T-lymphocytes): the percentage of CD95-DR3 ${ }^{+}$cells (a) and mean DR3 fluorescence intensity on their surface (b); the comparison of distribution density of DR3 receptor fluorescence on the surface of newly isolated CD95-DR3 ${ }^{+}$nCTLs with the parameter when cultured without activators added or with anti-DR3 mAbs (monoclonal antibodies) (c). AIM: acute infectious mononucleosis; IC: isotypical control; * statistically significant differences compared to healthy children $(p<0.05)$

Characteristic of nCTLs with CD95 ${ }^{+} D R 3^{+}$ phenotype in healthy children and children with AIM. AIM children compared to healthy children were found to have 1.6 decrease of $C D 95^{+} D R 3^{+}$cell number $(p=0.011)$ when $\mathrm{nCTLs}$ were stimulated by anti-CD95 antibodies. 1.7 increase of DR3 expression density on the surface of $\mathrm{CD}^{2} 5^{+} \mathrm{DR} 3^{+}$cells $(\mathrm{p}=0.004)$ was revealed in newly isolated nCTLs in children with AIM. In all other cases the differences were found neither in the content of CD95 ${ }^{+} \mathrm{DR}^{+}$cells, nor in the expression density of CD95 or DR3 on their surface (See Figure 4, Figure 7).

No differences were detected in the content of 


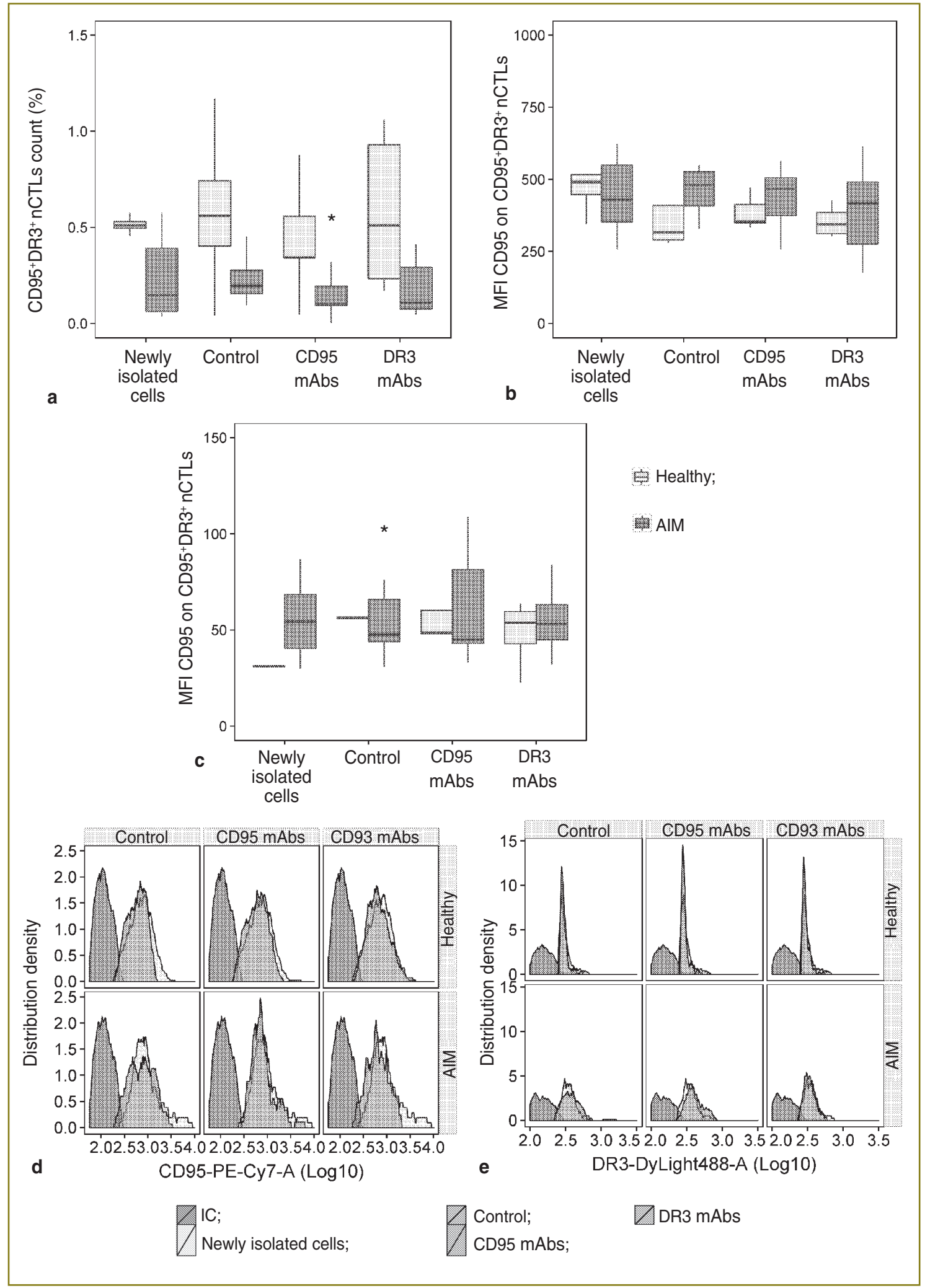

Figure 7. Characteristic of CD95+DR3 ${ }^{+} \mathrm{nCTLs}$ (naive cytotoxic T-lymphocytes): the percentage of CD95+DR3 ${ }^{+}$cells (a), mean CD95 fluorescence intensity (b) and mean DR3 fluorescence intensity (c) on their surface; the comparison of distribution density of CD95 receptor fluorescence on the surface of newly isolated $C D 95^{+} D R 3^{+} n C T L s$ with the parameter when cultured without activators added or with anti-CD95 or anti-DR3 mAbs (monoclonal antibodies) (d); the comparison of density distribution of DR3 receptor fluorescence on the surface of newly isolated $C D 95^{+} \mathrm{DR} 3^{+} \mathrm{nCTLs}$ with this parameter when cultured without activators added or with anti-CD95 or anti-DR3 mAbs (e). AIM: acute infectious mononucleosis; IC: isotypical control; * statistically significant differences compared to healthy children $(p<0.05)$ 
CD95+DR3 ${ }^{+}$cells and DR3 or CD95 expression density on their surface compared to newly isolated nCTLs regardless of nCTLs culture conditions of healthy children and children with AIM (See Figure 7).

A direct correlation was found between an apoptosis level and $C D 95^{+}{ }^{+} R 3^{+}$cell content only when $\mathrm{nCTLs}$ were cultured with anti-CD95 antibodies added in healthy children ( $r=0.88 ; p=0.049)$ and children with AIM $(r=0.73$; $\mathrm{p}=0.010$ ). No correlation was revealed between an apoptosis level and CD95 or DR3 expression density on the surface of $C D 95^{+} D R 3^{+}$cells in all cases under study.

Discussion. When studying $\mathrm{nCTLs}$ susceptibility to apoptosis in healthy children and children with AIM we assessed the expression of membrane receptors CD95 and DR3. Total nCTLs pool was divided into three phenotypic groups depending on the expression features of CD95 and DR3 receptors on their surface. We analyzed the contribution of each of the phenotypes in total apoptotic level of total nCTLs fraction.

An increased apoptotic level of total fraction of isolated $\mathrm{nCTLs}$ was detected in healthy children, both in the control and in those with specific activation of CD95 or DR3 receptors. Moreover, against the background of the increase, there was the decrease of the number of cell with phenotype CD95+DR3- only. CD95 receptor expression density on their surface decreased as well. It is our belief that in healthy children the main contribution in nCTLs apoptosis level is made by the subpopulation with phenotype CD95'DR3-, i.e. it is this subpopulation of naive T-lymphocytes which dies due first to apoptosis. The findings indicate that in healthy children CD95+DR3apoptosis induction of isolated nCTLs requires no participation of other immune competent cells, as well as additional stimulation of CD95 receptor.

In children with AIM, newly isolated nCTLs showed their apoptosis resistance that can be considered as a protective mechanism of a macroorganism aimed at nCTLs pool maintenance in the course of an antiviral immune response. Under specific culture, nCTLs in children with AIM lost their resistance to apoptosis in the control but persisted apoptosis resistance in CD95 receptor activation. It may be assumed that nCTLs resistance to apoptosis in AIM is due to CD95 receptor participation, and depends on the effect of other immune competent cells.

It should be noted that CD95 activation in AIM resulted in no changes of an apoptosis level of the total nCTLs pool compared to the newly isolated cells. In addition, there was the content decrease of T-lymphocytes with phenotype CD95'DR3-, and the increase of the number of cells with CD95-DR3- phenotype (data on nCTLs with CD95-DR3- phenotype have not been submitted). CD95 receptor binding on a cell membrane is known to result in its internalization [18]. Further signal transmission in AIM is expected to be absent, nCTLs apoptosis not developing. In addition, due to CD95 receptor internalization, cell phenotype changes.
Cells with CD95-DR3- phenotype potentially are more resistant to apoptosis induction with the receptors CD95 and DR3 involved. nCTLs resistance to apoptosis with CD95 activation is likely to be related to the changed balance of pro- and anti-apoptotic factors in these cells in AIM. So, in vitro studies [3] have demonstrated CD8 ${ }^{+}$ T-lymphocytes expressing CD95 receptor in EBV to manifest apoptosis resistance due to the expression increase of anti-apoptotic factor Bcl-2.

Cells with CD95'DR3- phenotype prevail quantitatively in a general $\mathrm{nCTLs}$ pool, while DR3-expressing cells (phenotypes CD95-DR3 ${ }^{+}$and $\mathrm{CD}^{-} 5^{+} \mathrm{DR}^{+}$) are present in small amounts. Compared to newly isolated nCTLs, there were observed no changes in the content of DR3-expressing cells regardless of culture conditions both in healthy children, and in children with AIM. In this respect, when evaluating the contribution of cell with this phenotype in total nCTLs apoptosis level it seems appropriate to consider them only as minor subpopulations participating in the control of apoptosis of CD95+DR3- cells.

In newly isolated cells, as well as in the control in children with AIM there was the decrease of the content of $\mathrm{CD}^{-} \mathrm{DR}^{+}$cells compared to healthy children. Moreover, DR3 receptor activation leveled the revealed differences. It should be noted that this fact is related to the decrease of this index in healthy children rather than to the increasing number of CD95-DR3 ${ }^{+} \mathrm{nCTLs}$ in children with AIM. Besides, in healthy children when DR3 receptor was activated, the number of CD95-DR3 ${ }^{+}$cells correlated directly to the apoptosis level of nCTLs pool. It can be assumed that in the norm apoptosis level in newly isolated $\mathrm{nCTLS}$ does not depend on the number of cells with $\mathrm{CD}^{-} 5^{-} \mathrm{DR}^{+}$phenotype. The correlation appears only under the conditions of DR3 receptor activation. In AIM a direct correlation of the parameters under study was initially found only in newly isolated cells, and was not detected in DR3 receptor activation. Thus, cells with CD95-DR3 ${ }^{+}$phenotype contribute differently to the total apoptosis level of nCTLs pool in healthy children and AlM children.

In our view, CD95+DR3 ${ }^{+}$nCTLs able to respond to the stimulation of both receptors are of particular interest. CD95 receptor activation results in the reduction of cell count with phenotype CD95+DR3 ${ }^{+}$ against the background of nCTLs apoptosis decrease in children with AIM compared to healthy children. In addition, in healthy children and in children with AIM the apoptosis level correlates directly to the content of $\mathrm{CD}^{+} 5^{+} \mathrm{DR} 3^{+}$cells. It is our belief that CD95 receptor in children with AIM participates in the apoptosis initiation of $\mathrm{CD}^{+} 5^{+} \mathrm{DR} 3^{+} \mathrm{T}$-lymphocytes. Despite an increased DR3 expression density on the surface of CD95+DR3 ${ }^{+}$ cells of newly isolated $\mathrm{nCTLs}$ in children with AIM, DR3 receptor activation results in neither the change of their number, nor nCTLs apoptosis level compared to healthy children. This fact indicates non-participation of DR3 
receptor in apoptosis initiation of $\mathrm{CD} 95^{+} \mathrm{DR} 3^{+} \mathrm{T}$-cells in healthy children and children with AIM. Thus, the stimulation of CD95 or DR3 receptors has a different effect on the susceptibility of CD95 ${ }^{+} D R 3^{+}$T-lymphocytes to apoptosis in AIM. In general, minor subpopulation of CD95+DR3 ${ }^{+}$cells makes an insignificant contribution to the total nCTLs apoptosis level in CD95 activation. The observed difference in nCTLs apoptosis level in healthy children and children with AIM is likely to be related to the potential capability of $\mathrm{CD} 95^{+} \mathrm{DR} 3^{+}$cells to modulate the susceptibility of CD95+DR3- T-lymphocytes to apoptosis.

Conclusion. The main contribution to the apoptosis of naive cytotoxic T-lymphocytes in healthy children is made by CD95+DR3- phenotype cells, while the cells with CD95-DR3 ${ }^{+}$and CD95 $^{+}$DR3 $^{+}$phenotype are supposed to perform a regulatory function. AIM in children is accompanied by decreased nCTLs sensitivity to apoptosis that is considered to be a favorable prognostic sign. DR3 expression on nCTLs surface in healthy children and children with AIM is different. However, DR3 receptor activation results in no change of nCTLs apoptosis level in children with AIM compared to healthy children. We think CD95-mediated signaling pathways in CD95+DR3- and CD95+DR3 ${ }^{+}$cells to participate in the realization of a mechanism of $\mathrm{nCTLs}$ resistance to apoptosis in AIM.

The evaluation of nCTLs susceptibility to CD95induced apoptosis can serve as a promising test to give additional characteristic of the state of a cell component of immune system in AIM.

Study Funding and Conflicts of Interest. The study was not funded by any sources, and the authors have no conflicts of interest related to the present study.

\section{References}

1. Scherrenburg J., Piriou E.R.W.A.N., Nanlohy N.M., van Baarle D. Detailed analysis of Epstein-Barr virus-specific CD4+ and $\mathrm{CD} 8^{+} \mathrm{T}$ cell responses during infectious mononucleosis. Clin Exp Immunol 2008; 153(2): 231-239, http://dx.doi.org/10.1111/ j.1365-2249.2008.03699.x.

2. Womack J., Jimenez M. Common questions about infectious mononucleosis. Am Fam Physician 2015; 91(6): 372-376.

3. Tanner J.E., Alfieri C. Epstein-Barr virus induces Fas (CD95) in T cells and Fas ligand in B cells leading to T-cell apoptosis. Blood 1999; 94(10): 3439-3444.

4. Walczak H., Krammer P.H. The CD95 (APO-1/Fas) and the TRAIL (APO-2L) apoptosis systems. Exp Cell Res 2000; 256(1): 58-66, http://dx.doi.org/10.1006/excr.2000.4840.

5. Papoff G., Hausler P., Eramo A., Pagano M.G., Di Leve G., Signore A., Ruberti G. Identification and characterization of a ligand-independent oligomerization domain in the extracellular region of the CD95 death receptor. J Biol Chem 1999; 274(53): 38241-38250, http://dx.doi.org/10.1074/jbc.274.53.38241.

6. Screaton G.R., Xu X.-N., Olsen A.L., Cowper A.E.,
Tan R., McMichael A.J., Bell J.I. LARD: a new lymphoid-specific death domain containing receptor regulated by alternative premRNA splicing. Proc Natl Acad Sci USA 1997; 94(9): 46154619, http://dx.doi.org/10.1073/pnas.94.9.4615.

7. Meylan F., Davidson T.S., Kahle E., Kinder M., Acharya K., Jankovic D., Bundoc V., Hodges M., Shevach E.M., Keane-Myers A., Wang E.C., Siegel R.M. The TNF-family receptor DR3 is essential for diverse T cell-mediated inflammatory diseases. Immunity 2008; 29(1): 79-89, http:// dx.doi.org/10.1016/j.immuni.2008.04.021.

8. Buchan S.L., Taraban V.Y., Slebioda T.J., James S., Cunningham A.F., Al-Shamkhani A. Death receptor 3 is essential for generating optimal protective $\mathrm{CD} 4^{+} \mathrm{T}$-cell immunity against Salmonella. Eur J Immunol 2012; 42(3): 580-588, http://dx.doi. org/10.1002/eji.201041950.

9. Klas C., Debatin K.M., Jonker R.R., Krammer P.H. Activation interferes with the APO-1 pathway in mature human $\mathrm{T}$ cells. Int Immunol 1993; 5(6): 625-630, http://dx.doi.org/10.1093/ intimm/5.6.625.

10. Alderson M.R., Armitage R.J., Maraskovsky E., Tough T.W., Roux E., Schooley K., Ramsdell F., Lynch D.H. Fas transduces activation signals in normal human $\mathrm{T}$ lymphocytes. J Exp Med 1993; 178(6): 2231-2235, http://dx.doi.org/10.1084/ jem.178.6.2231.

11. Utkin O.V., Novikov V.V. Death receptors in modulation of apoptosis. Uspekhi sovremennoi biologii 2012; 132(4): 381390.

12. Brown I.E., Mashayekhi M., Markiewicz M., Alegre M.L., Gajewski T.F. Peripheral survival of naive CD8 ${ }^{+}$ T cells apoptosis. Apoptosis 2005; 10(1): 5-11, http://dx.doi. org/10.1007/s10495-005-6056-9.

13. Balfour H.H. Jr., Odumade O.A., Schmeling D.O., Mullan B.D., Ed J.A., Knight J.A., Vezina H.E., Thomas W., Hogquist K.A. Behavioral, virologic, and immunologic factors associated with acquisition and severity of primary Epstein-Barr virus infection in university students. J Infect Dis 2013; 207(1): 80-88, http://dx.doi.org/10.1093/infdis/jis646.

14. Mao J.Q., Yang S.L., Song H., Zhao F.Y., Xu X.J., Gu M.E., Tang Y.M. Clinical and laboratory characteristics of chronic active Epstein-Barr virus infection in children. Zhongguo Dang Dai Er Ke Za Zhi 2014; 16(11): 1081-1085.

15. Skaletskaya A., Bartle L.M., Chittenden T., McCormickA.L., Mocarski E.S., Goldmacher V.S. A cytomegalovirus-encoded inhibitor of apoptosis that suppresses caspase-8 activation. Proc Natl Acad Sci USA 2001; 98(14): 7829-7834, http:lldx.dol. org/10.1073/pnas.141108798.

16. Seirafian S., Prod'homme V., Sugrue D., Davies J., Fielding C., Tomasec P., Wilkinson G.W. Human cytomegalovirus suppresses Fas expression and function. J Gen Virol 2014; 95(Pt 4): 933-939, http://dx.doi.org/10.1099/ vir.0.058313-0.

17. Twohig J.P., Marsden M., Cuff S.M., Ferdinand J.R., Gallimore A.M., Perks W.V., Al-Shamkhani A., Humphreys I.R., Wang E.C. The death receptor 3/TL1A pathway is essential for efficient development of antiviral CD4 $4^{+}$and $\mathrm{CD}^{+}{ }^{+} \mathrm{T}$-cell immunity. FASEB J 2012; 26(8): 3575-3586, http://dx.doi. org/10.1096/fj.11-200618.

18. Schneider-Brachert W, Heigl U., Ehrenschwender M. Membrane trafficking of death receptors: implications on signalling. Int J Mol Sci 2013, 14(7): 14475-14503, http://dx.doi. org/10.3390/ijms 140714475. 